\title{
Salage de l'Emmental \\ I. Influence des conditions de saumurage sur l'absorption du sel et sa cinétique
}

\author{
J.F. CHAMBA \\ Institut Technique du Gruyère, Pré Germain, 74801 La Roche-sur-Foron Cedex, France
}

\section{Résumé}

L'absorption du sel a été mesurée indirectement en observant la quantité de sel qui passe de la saumure au fromage. La vitesse d'absorption du sel décroît au cours du saumurage, elle varie, exprimée en $\mathrm{g} \cdot \mathrm{h}^{-1}$ par $100 \mathrm{~kg}$ de fromage, de 22,8 à $10 \mathrm{~h}, 18,9$ à $20 \mathrm{~h}, 16,4$ à $30 \mathrm{~h}, 14,0$ à $50 \mathrm{~h}, 12,4$ à $70 \mathrm{~h}$ et 10,8 à $96 \mathrm{~h}$. La quantité de sel absorbée est, en moyenne, par $100 \mathrm{~kg}$ de fromage, de $709,5 \mathrm{~g}$ en $48 \mathrm{~h}$, de $903 \mathrm{~g}$ en $72 \mathrm{~h}$ et de $1039 \mathrm{~g}$ en $96 \mathrm{~h}$ avec des saumures fraîchement préparées d'une concentration moyenne de $265 \mathrm{~g} \cdot \mathrm{l}^{-1}$ et à $12-13^{\circ} \mathrm{C}$. Les pertes de poids du fromage augmentent avec la durée du saumurage : $0,94 \%$ en $48 \mathrm{~h}, 1,17 \%$ en $72 \mathrm{~h}$ et $1,33 \%$ en $96 \mathrm{~h}$.

Par comparaison à une saumure à $13{ }^{\circ} \mathrm{C}$, la quantité de sel absorbée est de $78,2 \%$ à $8{ }^{\circ} \mathrm{C}$ et de $118 \%$ à $18{ }^{\circ} \mathrm{C}$. A $12-13^{\circ} \mathrm{C}$, avec des saumures dont la concentration en sel variait de $204 \mathrm{~g} \cdot 1^{-1}$ à $318 \mathrm{~g} \cdot 1^{-1}$, nous avons obtenu la relation suivante : sel absorbé en $48 \mathrm{~h}$ (g pour $100 \mathrm{~kg}): 3,79$ concentration saumure $\left(\mathrm{g} \cdot \mathrm{I}^{-1}\right)-294,5$ avec un coefficient de corrélation de $0,978^{* *}$. Le $\mathrm{pH}$ de la saumure, compris entre 5,2 et 7 , n'a aucune influence sur l'absorption du sel. Lorsque la saumure est constituée par dilution du sel, soit dans du lactosérum d'égouttage d'Emmental, soit dans un mélange pour moitié d'eau et de lactosérum pour simuler le vieillissement des saumures, l'absorption du sel est fortement réduite. Enfin, nous avons obtenu une corrélation positive $\left(\mathrm{r}=0,85^{* *}\right)$ entre l'humidité de la zone corticale du fromage et la quantité de sel absorbée.

Mots clés : Emmental - Saumurage - Absorption du sel - Cinétique.

\section{Summary}

Emmental cheese salting. I. Brining conditions effects on salt absorption and its kinetic

The salt absorption has been indirectly measured by observation of the salt amount absolute variation which is due to the salt migration from the brine to the cheese. The rate of the salt absorption decreases during brining, it varies - expressed in $\mathrm{g} \cdot \mathrm{h}^{-1}$ for $100 \mathrm{~kg}$ of cheese - from 22.8 after 10 hours, 16.4 after 30 hours, 14.0 after 50 hours, 12.4 after 70 hours to 10.8 after 96 hours. The mean of the salt absorption for $100 \mathrm{~kg}$ of cheese is $709.5 \mathrm{~g}$ after 48 hours, $903 \mathrm{~g}$ after 72 hours and $1039 \mathrm{~g}$ after 96 hours in 
newly made brine at $13{ }^{\circ} \mathrm{C}$ and with a concentration of $265 \mathrm{~g} \cdot 1^{-1}$. The cheese weight losses increase with brining time : $0.94 \%$ after 48 hours, $1.17 \%$ after 72 hours and 1.33 after 96 hours.

Comparatively to a brine at $13{ }^{\circ} \mathrm{C}$, the absorbed salt amount is $78.2 \%$ at $8{ }^{\circ} \mathrm{C}$ and $118 \%$ at $18{ }^{\circ} \mathrm{C}$. At $12-13{ }^{\circ} \mathrm{C}$, with brines which the salt concentration varying between $204 \mathrm{~g} \cdot \mathrm{I}^{-1}$ and $318 \mathrm{~g} \cdot \mathrm{I}^{-1}$, we reached the following relationship : salt absorbed after 48 hours (g per $100 \mathrm{~kg}$ ) : 3.79 brine concentration $\left(\mathrm{g} \cdot 1^{-1}\right)-294.5$, the coefficient of correlation is $0.978^{* *}$. The brine $\mathrm{pH}$, between 5.2 and 7 , has no effect on the salt absorption. When the brine is made by dilution of salt in whey or in a mixture composed by half water and half whey to simulate the ageing of brines, the salt absorption is highly reduced. Finally, we reached a positive correlation $\left(\mathrm{r}=0,85^{* *}\right)$ between the moisture of the cheese rind zone and the salt amount absorbed.

Key words : Emmental cheese - Brining - Salt absorption - Salting kinetic.

\section{Introduction}

Kosikowski et Mocquot (1958) considèrent que le salage est l'une des six étapes fondamentales de la fabrication des fromages, aussi bien « dans le désert du Sahara par les tribus nomades que dans les usines garnies de carreaux de faïence et de matériel en acier inoxydable d'Europe ou des EtatsUnis ». Traditionnellement, c'est une opération classique dont les règles ont été fixées par l'expérience compte tenu des moyens matériels existants. Il en est du salage comme de nombreuses autres phases de la fabrication. Le salage par saumurage apparaît comme une opération simple dans l'élaboration de l'Emmental qui n'a guère évolué depuis longtemps (Munier, 1858 ; Martin, 1898). On peut retenir trois grands rôles joués par le salage pour tous les fromages (ALAIS, 1975).

- Complément d'égouttage se traduisant par une poursuite de l'exsudation de lactosérum, cette action qui est particulièrement nette à la surface du fromage participe à la formation de la croûte.

- Rendre le fromage plus sapide, le sel qui est par lui-même une des quatre saveurs de base joue également un rôle d'exhausteur du goût des produits sapides.

- Agir sur le développement des bactéries du fromage, soit à la surface, soit dans la pâte, en abaissant l'activité de l'eau $\left(\mathrm{a}_{\mathrm{w}}\right)$, le sel est un facteur puissant de sélection bactérienne et de l'activité des enzymes.

Le salage en saumure est une opération constamment utilisée dans tous les pays lors de la fabrication de l'Emmental. Cependant, bien que de nombreux auteurs de plusieurs pays publient sur la fabrication de ce fromage, le salage a été peu étudié. Toutefois, la modernisation de la technique de saumurage (mécanisation, régulation des caractéristiques de la saumure) exige que le mécanisme de l'absorption du sel et l'influence des divers paramètres (température et composition de la saumure par exemple), soient bien connus. De plus, les consommateurs demandent des produits de meilleure qualité, plus réguliers dont la composition et les caractéristiques organoleptiques puissent être garanties. Ainsi, la demande d'une plus grande maîtrise de la technologie et de la qualité de l'Emmental exige également une meilleure connaissance du 
salage, c'est la raison de ce travail. Nous avons voulu répondre aux questions suivantes :

- A quelle vitesse le sel est-il absorbé par l'Emmental, quelle est la cinétique du salage, quelles sont les teneurs en sel que l'on peut attendre en faisant varier la durée du saumurage de l'Emmental? KunManN et al. (1976) ont montré que le doublement de la durée du saumurage de 2 à 4 jours augmente de $30 \%$ la teneur en sel. Bergere et al. (1969) ont montré qu'avec un saumurage de 96 heures et en apportant fréquemment (deux fois par semaine) du sel sur la croûte tout au long de l'affinage, on peut presque doubler la teneur de l'Emmental en sel. Par contre, il n'existe aucune donnée sur la cinétique de l'absorption du sel.

- Quelle est l'influence de la température et de la composition de la saumure ( $\mathrm{pH}$, acidité, concentration) sur l'absorption? Cette étude a déjà été effectuée pour de nombreux fromages à pâte molle ou à pâte pressée (HARDY, 1976 ; HARdY, 1983 ; JAKUBOWSKI, 1968 ; GeurTS et al., 1974 ; MANSOUR, 1972 ; Terre et al., 1985). Pour ces fromages, il est admis que la concentration en sel de la saumure a une influence directe sur la teneur en sel du fromage. En Emmental, les observations de Kurmann et al. (1976) et de RothenbuHLer (1970) montrent que les températures élevées accroissent la teneur en sel mais soit ils utilisent des températures qui ne se rencontrent pas en pratique $\left(1^{\circ} \mathrm{C}\right.$, $4{ }^{\circ} \mathrm{C}, 30^{\circ} \mathrm{C}$ ), soit les observations n'ont été effectuées que sur des petits morceaux de fromage (RoTHENBUHLER, 1970). Une meilleure connaissance de l'influence de ces facteurs peut donner lieu à des applications pratiques : consignes à donner aux fromageries, conception des installations de saumurage (régulation des paramètres).

\section{Matériel et méthodes}

\section{A. Mesure de la cinétique de l'absorption du sel}

La principale difficulté pour étudier la cinétique de l'absorption du sel par l'Emmental tient à ses dimensions : 0,72 à $0,78 \mathrm{~m}$ de diamètre sur 0,14 à $0,18 \mathrm{~m}$ d'épaisseur. De ce fait, la répartition du sel est très hétérogène comme l'ont déjà montré Uotila (1965) en Finlande, MaIr-waldburg et Heinrich (1966) en Bavière, BERgere et al. (1969) en France, ainsi que Kurmann et al. (1976) en Suisse. Cette hétérogénéité dans la répartition du sel après le saumurage a également été observée par LE GraET et al. (1986) sur le fromage de Beaufort où ils trouvent, exprimé en $\mathrm{g}$ de $\mathrm{Na}$ par $\mathrm{kg}$ de fromage, $2,95 \mathrm{~g}$ dans les $3 \mathrm{~mm}$ de croûte, $2,78 \mathrm{~g}$ dans la partie sous croûte $(17 \mathrm{~mm})$ et $0,55 \mathrm{~g}$ au centre du fromage. Pour obtenir un échantillon représentatif de la teneur en sel de ce fromage, il faut le découper entièrement afin de prélever un secteur représentant exactement la proportion pondérale de la zone centrale et de la zone corticale. Or, le calcul montre que sur un échantillon de $1 \mathrm{~kg}$ (secteur de 4,8 : L 0,36 m, $10,16 \mathrm{~m}$, épaisseur en talon $0,03 \mathrm{~m}$ ) une erreur de découpage de $1 \mathrm{~mm}$ conduit à une différence de $\pm 75 \mathrm{~g}$ de la teneur en sel de l'Emmental soit \pm 10 à $15 \%$. L'observation directe de l'absorption du sel au cours du saumurage par son dosage dans le fromage serait donc très coûteuse 
et ne permet pas d'améliorer la précision des mesures. Cela nous a conduit à retenir une méthode d'observation indirecte : mesurer la qualité de sel qui passe de la saumure au fromage.

\section{Appareil de saumurage}

Il est constitué par :

- Un bac cylindrique (diamètre $=0,90 \mathrm{~m}, \mathrm{~h}=0,35 \mathrm{~m}$ ) en acier inoxydable (NSMC 316), dans lequel est immergé le fromage à saler, et un petit bac rectangulaire $(\mathrm{L}=0,30 \mathrm{~m}, 1=0,20 \mathrm{~m}, 0,40 \mathrm{~m})$ du même acier inoxydable contenant l'évaporateur du groupe frigorifique (serpentin); ils sont munis d'un couvercle.
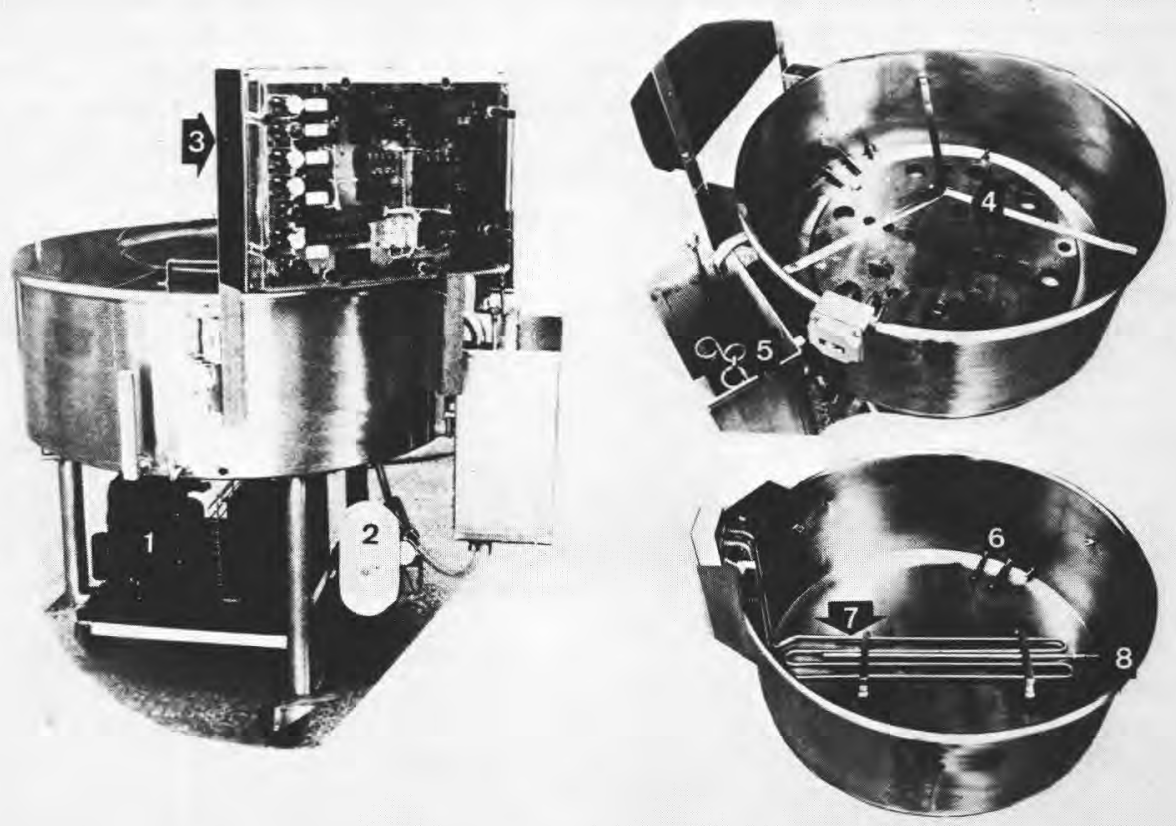

FIG. 1

Appareil de saumurage.

1. Groupe frigorifique. 2. Pompe de circulation. 3. Tableau électrique. 4. Croisillon de maintien. 5. Evaporateur. 6. Sondes des thermostats. 7. Résistance chauffante. 7. Rampe perforée.

\section{Brining machine.}

1. Refrigerator set. 2. Circulation pump. 3. Electric box. 4. Keep crosspiece. 5. Cold evaporator. 6. Thermostats probes. 7. Heating resistance. 8. Perfored tube. 
- Une pompe de circulation à engrenages en téflon débitant $220 \mathrm{~A} \cdot \mathrm{h}^{-1}$. L'aspiration se fait au fond du bac rectangulaire à travers une crépine et le refoulement s'effectue à travers une rampe située au fond du bac cylindrique. Par débordement, la saumure s'écoule dans le petit bac rectangulaire.

- Un groupe frigorifique et une résistance chauffante fixée au fond du bac sont commandés chacun par un thermostat.

- Un tableau électrique étanche regroupant l'ensemble des appareils de commande et de sécurité. La commande de la pompe servant d'interrupteur général.

Cet appareil a été construit par les Etablissements Chalon-Mégard à La Cluse - Nantua (01).

Dans le bac cylindrique, le fromage est maintenu immergé par un croisillon et la saumure circule en permanence. Le fonctionnement indépendant du groupe frigorifique et de la résistance chauffante assure un maintien de la température à $\pm 0,5^{\circ} \mathrm{C}$.

\section{Préparation de la saumure}

Elle est préparée indépendamment dans un bac en polyéthylène avec du sel épuré (sulfate $\leqslant 0,012 \%$ ) et de l'eau potable du réseau ( $\mathrm{pH}: 7,4$; TA : 0 ; TA : $25 \mathrm{meq}$, soit $125^{\circ} \mathrm{F}$; dureté : $30-32{ }^{\circ} \mathrm{F}$ ). En règle générale, nous préparons une saumure saturée et la concentration désirée est obtenue par dilution. L'ajustement du $\mathrm{pH}$ est effectué soit à l'acide lactique concentré $(90 \%)$, soit avec de la lessive de soude. Nous avons utilisé des saumures fraîches ou n'ayant servi qu'un petit nombre de fois (3 à 5).

Dans les expériences ayant pour but d'étudier l'influence de l'usure de la saumure et donc pour simuler l'âge de la saumure, nous l'avons préparée avec un lactosérum d'égouttage d'Emmental récolté après les deux premières heures de pressage. La proportion d'eau et de sérum reproduisait divers degrés d'usure. Les caractéristiques des saumures seront décrites dans les résultats. Il faut de 1101 à 1201 de saumure, selon le poids du fromage saumuré pour assurer le remplissage normal de l'appareil, soit 1,4 à 1,7 I par $\mathrm{kg}$ de fromage.

\section{Mesure de la quantité de sel absorbé}

La saumure introduite dans l'appareil est pesée. La température et le $\mathrm{pH}$ sont ajustés si nécessaire et un échantillon de saumure est prélevé. Le fromage pesé est immergé et les prélèvements sont ensuite effectués environ toutes les dix heures.

Après saumurage, le fromage est retiré de la saumure, égoutté et pesé. L'appareil est complètement vidangé et la saumure recueillie est pesée, un échantillon est prélevé. Le poids des échantillons prélevés est ajouté au poids final de la saumure. Après la pesée et le dosage des chlorures, nous disposons des éléments suivants :

- poids du fromage avant et après saumurage,

- poids de sel avant et après saumurage du fromage,

- concentration de la saumure lors des divers prélèvements. 
Ces éléments nous permettent de calculer :

- le poids de sel absorbé par le fromage et pour $100 \mathrm{~kg}$ de fromage salé,

- la proportion de sel absorbé entre chacun des prélèvements est obtenue par proportionalité avec l'abaissement de la concentration de la saumure. $\mathrm{Ce}$ mode de calcul admet que l'exsudation de la phase aqueuse du fromage qui, avec l'absorption du sel, contribue à l'abaissement de la concentration de la saumure est linéaire. Ce point sera examiné plus loin.

La précision de la balance utilisée pour peser les fromages et la saumure est de $0,05 \mathrm{~kg}$. L'erreur maximale ou incertitude absolue sur les pertes de poids du fromage est donc de $0,1 \mathrm{~kg}$ (pesée sans foncet). Pour l'évolution du poids de la saumure, l'incertitude absolue est de $0,2 \mathrm{~kg}$ (pesée dans un bac taré).

\section{Les fromages utilisés pour l'étude de l'absorption du sel}

Ils proviennent tous de la fromagerie de l'Ecole Nationale des Industries du Lait et des Viandes de La Roche-sur-Foron, et sont fabriqués soit en cuve multiple (4 fromages), soit en cuve unitaire et pressés en moules métalliques.

\section{B. Méthodes analytiques}

\section{Dosage du $\mathrm{NaCl}$}

La titration des chlorures dans le fromage avec une électrode à l'ion $\mathrm{Ag}^{+}$ a été proposée dès 1969 par Boy et a été retenue comme méthode officielle française (1980). C'est cette méthode titrimétrique que nous avons également utilisée pour doser le $\mathrm{NaCl}$ dans la saumure.

Nous obtenons un écart type de répétabilité de $0,037 \mathrm{~g} \cdot \mathrm{k}^{-1}$ avec la saumure et de $0,0477 \mathrm{~g} \cdot \mathrm{kg}^{-1}$ avec le fromage. Ainsi, la répétabilité pour le dosage du $\mathrm{NaCl}$ (écart entre deux déterminations ayant une probabilité de $95 \%$ de ne pas être dépassée) est de $0,1 \mathrm{~g} \cdot \mathrm{kg}^{-1}$ pour la saumure et de $0,13 \mathrm{~g} \cdot \mathrm{kg}^{-1}$ pour le fromage (GRAPPIN, 1976).

La teneur en sel de la saumure est mesurée sur un volume connu $(10 \mathrm{ml})$ qui est également pesé. Le résultat obtenu peut donc être calculé en $\mathrm{g}$ par $\mathrm{kg}$ ou en g par l. Puisque la saumure est pesée lors des essais, nous utilisons la teneur en $\mathrm{g} \cdot \mathrm{kg}^{-1}$ pour effectuer les calculs d'absorption. Cependant l'analyse donnant également la teneur en $\mathrm{g} \cdot \mathrm{I}^{-1}$, c'est cette expression plus courante que nous utiliserons dans le texte.

\section{Extrait sec-total du fromage}

Cinq grammes de fromage râpé sont pesés exactement dans une coupelle en acier inoxydable puis desséchés par passage dans une étuve ventilée à $103^{\circ} \mathrm{C} \pm 1{ }^{\circ} \mathrm{C}$ pendant 15 heures. Après refroidissement au dessiccateur garni d'un agent déshydratant, la capsule contenant le fromage est pesée à nouveau. L'extrait sec total (EST) est exprimé en g pour $100 \mathrm{~g}$ de fromage. La nature des échantillons de fromage est indiquée avec les résultats. 
3. Acidité et $\mathrm{pH}$ de la saumure

L'acidité est titrée à la soude $\mathrm{N} / 9$ et exprimée en ${ }^{\circ}$ Dornic $\left(1^{\circ} \mathrm{D}=0,1 \mathrm{ml}\right.$ de $\mathrm{NaOH}$ N/9). Le pH est obtenu à $20^{\circ} \mathrm{C}$ avec une électrode combinée et un $\mathrm{pH}$ mètre PHN 75 de TACUSSEL.

\section{Résultats}

\section{A. Variabilité de l'absorption du sel par l'Emmental}

Dès les premières mesures, nous avions observé des différences d'absorption du sel dans des conditions très voisines de saumurage. Le tableau 1 met en évidence les écarts obtenus avec deux modalités de salage (concentration de la saumure et durée du saumurage).

TABLEAU 1

Variabilité de l'absorption du $\mathrm{NaCl}$

$\mathrm{NaCl}$ absorption variability

\begin{tabular}{c|c|c|c|c}
\hline Essais & $\begin{array}{c}\text { Concentration } \\
\text { de la saumure } \\
\mathrm{g} \cdot 1^{-1}\end{array}$ & $\begin{array}{c}\text { Durée du } \\
\text { saumurage } \\
\mathrm{h}\end{array}$ & $\begin{array}{c}\mathrm{NaCl} \text { absorbé } \\
\mathrm{g} / 100 \mathrm{~kg}\end{array}$ & $\begin{array}{c}\text { Pertes de poids } \\
\%\end{array}$ \\
\hline A 1 & 284 & 48 & 1028 & 1,32 \\
A 2 & 278 & 48 & 770 & 0,88 \\
A 3 & 274 & 48 & 705 & 0,97 \\
A 4 & 270 & 48 & 665 & 0,96 \\
B 1 & 255 & 72 & 587 & 1,2 \\
B 2 & 256 & 72 & 757 & 0,98 \\
B 3 & 255 & 72 & 723 & 1,05 \\
B 4 & 247 & 72 & 971 & 1,34 \\
\hline
\end{tabular}

Ces résultats peuvent être expliqués de deux façons :

- l'erreur expérimentale: l'addition des incertitudes absolues (pesées + dosages) conduit à une erreur maximale $(\mathrm{P} \geqslant 0,95)$ de 60 à $72 \mathrm{~g}$ de sel absorbés par fromage selon la concentration de la saumure. Les écarts de résultats observés sur les fromages $\mathrm{A} 3$ et $\mathrm{A} 4$ ou $\mathrm{B} 2$ et $\mathrm{B} 3$ se situent donc dans les limites de cette erreur maximale ;

- les caractéristiques individuelles des fromages expérimentaux : la variabilité des pertes de poids montre clairement qu'il y a un « effet fromage». Cette variabilité avait déjà été observée d'un jour à l'autre par MAIRWaldburg et HeinRich (1966). C'est également une difficulté rencontrée avec le Gouda par GeurTs et al. (1974) et qu'ils ont résolu en entreposant plusieurs 
jours les fromages à température de saumurage, afin de stabiliser la répartition de l'eau du fromage. Nous n'avons pas utilisé ce moyen car ces conditions nous éloignaient de celles rencontrées dans la pratique.

\section{B. Influence de l'EST du fromage}

L'une des caractéristiques primordiales est l'humidité de la zone corticale du fromage. Pour en mesurer l'influence, nous avons prélevé un échantillon à mi-rayon sur 9 fromages avant salage à l'aide d'une lame d'acier souple et aiguisée. Après découpage, nous obtenons un échantillon parallélépipédique de $2 \mathrm{~cm}$ d'épaisseur et de $5 \times 5 \mathrm{~cm}$ comprenant la croûte. Après saumurage, un échantillon identique est prélevé à proximité du premier prélèvement. Les résultats obtenus figurent dans le tableau 2.

\section{TABLEAU 2}

Evolution de la teneur en sel et de l'EST de la zone corticale $(0-2 \mathrm{~cm})$ lors du saumurage

Salt content and dry matter evolution in rind zone $(0-2 \mathrm{~cm})$ during brining

\begin{tabular}{c|c|c|c|c}
\hline \multirow{2}{*}{ Fromage } & \multicolumn{2}{|c|}{ Avant saumurage } & \multicolumn{2}{c}{ Après saumurage } \\
\cline { 2 - 5 } & $\mathrm{NaCl} \mathrm{g} / \mathrm{kg}$ & EST \% & $\mathrm{NaCl} \mathrm{g} / \mathrm{kg}$ & EST \% \\
\hline CM 1 & 1,04 & 62,55 & 15,19 & 65,40 \\
CM 2 & 1,03 & 62,20 & 17,21 & 66,90 \\
CM 3 & 0,87 & 62,60 & 13,36 & 65,60 \\
CM 4 & 0,87 & 62,50 & 13,36 & 65,80 \\
CM 5 & 1,63 & 65,10 & 13,79 & 66,50 \\
CM 6 & 1,84 & 63,00 & 13,70 & 64,05 \\
CT 1 & 1,07 & 60,90 & 22,50 & 64,00 \\
CT 2 & 1,02 & 63,50 & 12,33 & 65,70 \\
CT 3 & 0,85 & 60,10 & 23,70 & 62,30 \\
\hline Moyenne & 1,13 & 62,50 & 16,12 & 65,10 \\
\hline
\end{tabular}

A l'examen de ces résultats, on constate, outre l'augmentation des teneurs en sel de la zone corticale, l'augmentation de l'EST, en moyenne $+2,6$ points, de la zone des deux premiers centimètres sous croûte. Cette évolution explique la formation de la croûte produite par le saumurage, ainsi que les pertes de poids. La relation entre l'augmentation de la teneur en sel et l'humidité de la zone corticale du fromage est illustrée dans la figure 2. Ainsi, plus un fromage est humide, en particulier dans la zone corticale, plus il absorbe le sel au cours du saumurage. Cette dessiccation peut expliquer les différences d'absorption que nous avons observées entre les Emmentals saumurés dans des conditions très voisines. 


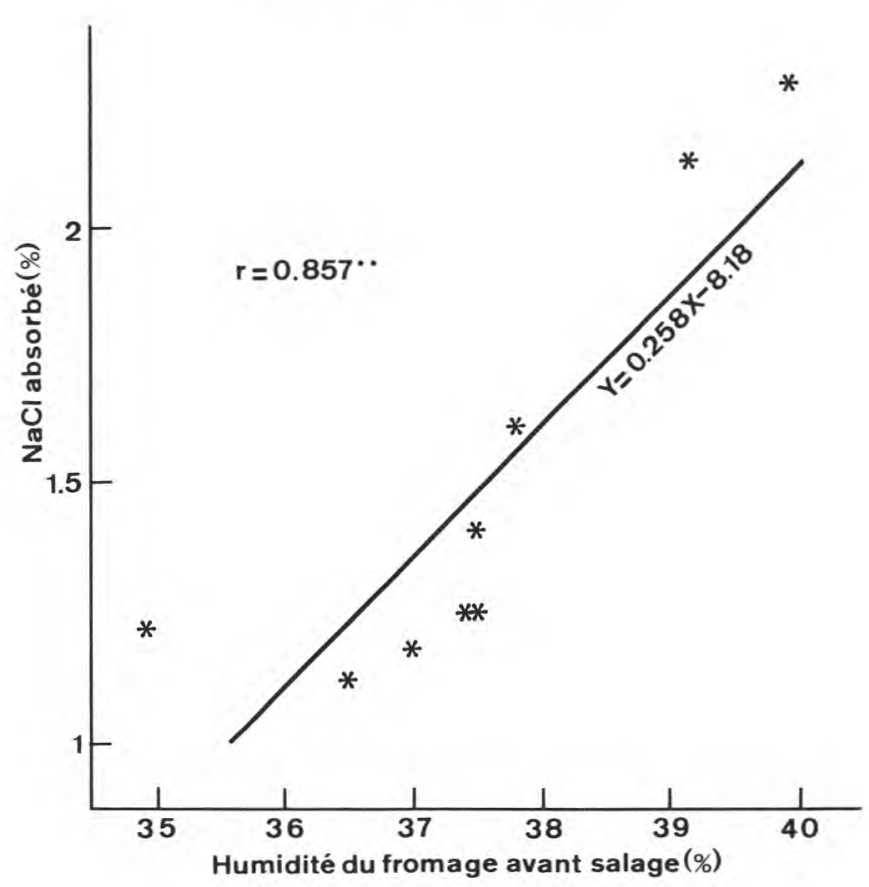

FIG. 2

Relation entre l'humidité de la zone corticale $(0-2 \mathrm{~cm})$ avant salage et l'absorption du sel. Relationship between rind zone $(0-2 \mathrm{~cm})$ moisture before brining and salt absorption.

\section{Influence de la durée de saumurage}

En France, le saumurage dure habituellement $48 \mathrm{~h}$ et parfois $72 \mathrm{~h}$; exceptionnellement, on observe des durées de $96 \mathrm{~h}$, ce sont donc ces durées qui ont été étudiées. Nous avons utilisé tous les résultats obtenus avec des saumurages à $12-13^{\circ} \mathrm{C}$ quels que soient la concentration ou le $\mathrm{pH}$ de la saumure (à l'exclusion des saumures contenant du lactosérum). La cinétique de l'absorption est représentée dans la figure 3. En prenant comme base de l'indice 100, la quantité de sel absorbée à $48 \mathrm{~h}$, et en utilisant les valeurs obtenues pour chacune des durées de salage, on obtient les résultats moyens présentés dans le tableau 3.

Ainsi, en ne saumurant que $24 \mathrm{~h}$, la quantité de sel absorbée serait d'environ $60 \%$ de celle obtenue avec un saumurage de $48 \mathrm{~h}$. Si l'on augmente celui-ci de 24 h (soit $+50 \%$ ), on accroît d'environ $30 \%$ la teneur en sel de l'Emmental et un doublement du temps de saumurage augmente de $60 \%$ l'absorption. En effet, comme le montre la figure 3, la vitesse d'absorption du sel décroît au cours du saumurage. Ainsi, à la moitié de la durée du saumurage, ce sont $60 \%, 62,6 \%$ et $61,9 \%$ du sel qui sont absorbés lorsque les fromages sont salés respectivement 48 h, 72 h et 96 h. 
TABLEAU 3

Influence de la durée de saumurage sur l'absorption du $\mathrm{NaCl}$ (valeurs relatives) Effect of brining time on $\mathrm{NaCl}$ absorption (relative values)

\begin{tabular}{c|c|c|c}
\hline \multirow{2}{*}{ Absorption } & \multicolumn{3}{|c}{ Saumurage de } \\
\cline { 2 - 4 } & $48 \mathrm{H}$ & $72 \mathrm{H}$ & $96 \mathrm{H}$ \\
\hline \multirow{2}{*}{ à $24 \mathrm{H}$} & 60 & 59 & 64,5 \\
à $48 \mathrm{H}$ & 100 & 100 & 100 \\
à $72 \mathrm{H}$ & - & 127,7 & 135 \\
à 96 H & - & - & 161,5 \\
\hline
\end{tabular}

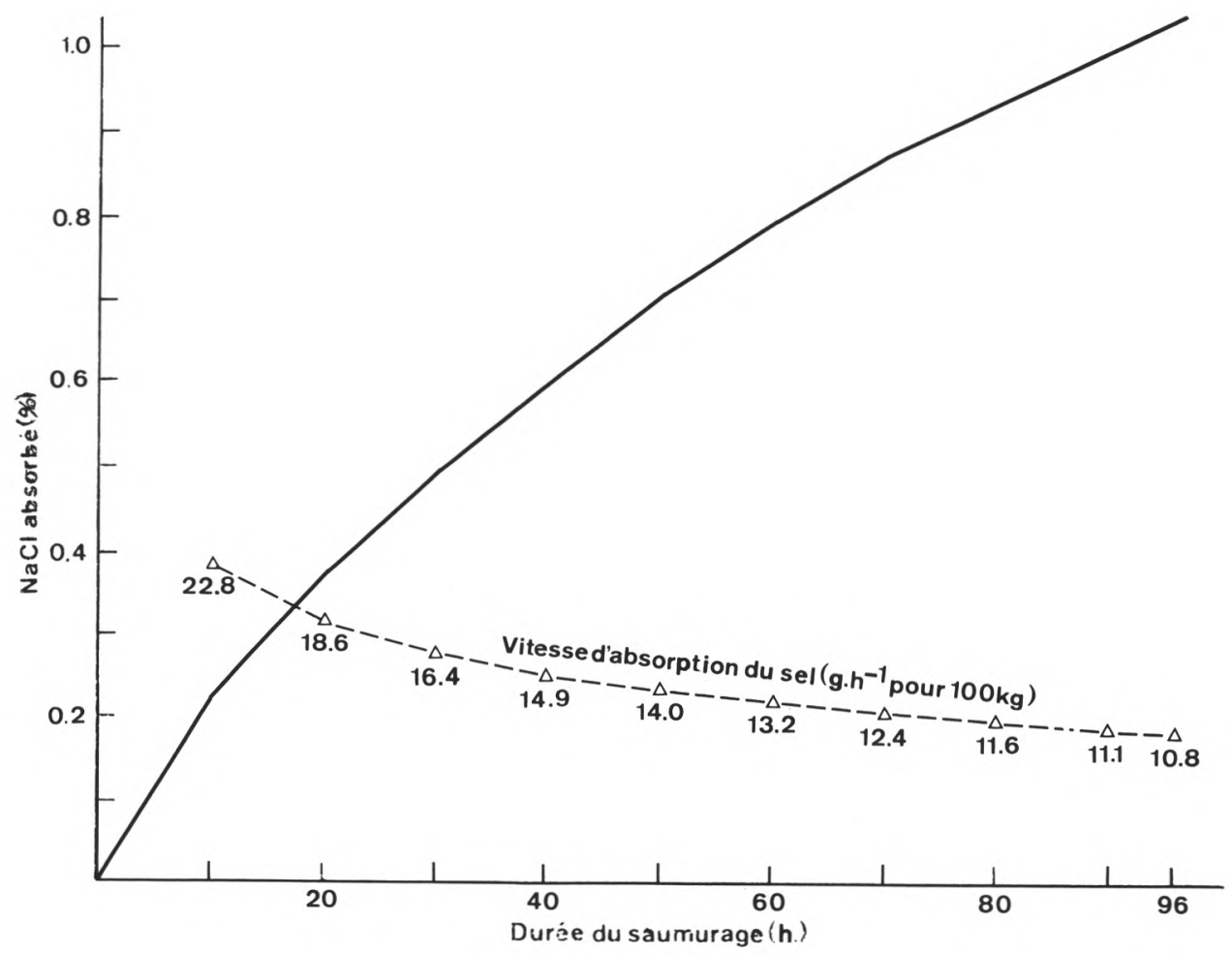

FIG. 3

Cinétique de l'absorption du sel par l'Emmental.

Kinetic of Emmental cheese salt absorption. 
Les pertes de poids apparentes du fromage augmentent également avec la durée du saumurage puisqu'elles sont en moyenne de $0,94 \%$ à $48 \mathrm{~h}$, de $1,17 \%$ à $72 \mathrm{~h}$ et $1,33 \%$ en $96 \mathrm{~h}$. Les échanges entre la saumure et le fromage se poursuivent donc activement, au moins jusqu'à $96 \mathrm{~h}$. On peut considérer que le fromage perd sous forme de phase aqueuse $1,65 \%$ en $48 \mathrm{~h}, 2,07 \%$ en $72 \mathrm{~h}$ et $2,37 \%$ en $96 \mathrm{~h}$ (sel absorbé + pertes de poids apparentes). Cet échange se traduit par une augmentation de l'EST du fromage dans la zone corticale comme cela a été montré précédemment.

\section{Influence de la température de la saumure}

Les températures de saumurage comparées dans le tableau 4 et illustrées par la figure 4 sont $8{ }^{\circ} \mathrm{C}, 13{ }^{\circ} \mathrm{C}$ et $18{ }^{\circ} \mathrm{C}, 5$ essais ont été effectués pour chaque température. On voit que la température de la saumure a un effet sur l'absorption du sel par l'Emmental. Ainsi, une augmentation de la température de la saumure de $13{ }^{\circ} \mathrm{C}$ à $18{ }^{\circ} \mathrm{C}$ augmente de $18 \%$ l'absorption du sel, alors qu'un abaissement de $13{ }^{\circ} \mathrm{C}$ à $8{ }^{\circ} \mathrm{C}$ la réduit de $21,8 \%$.

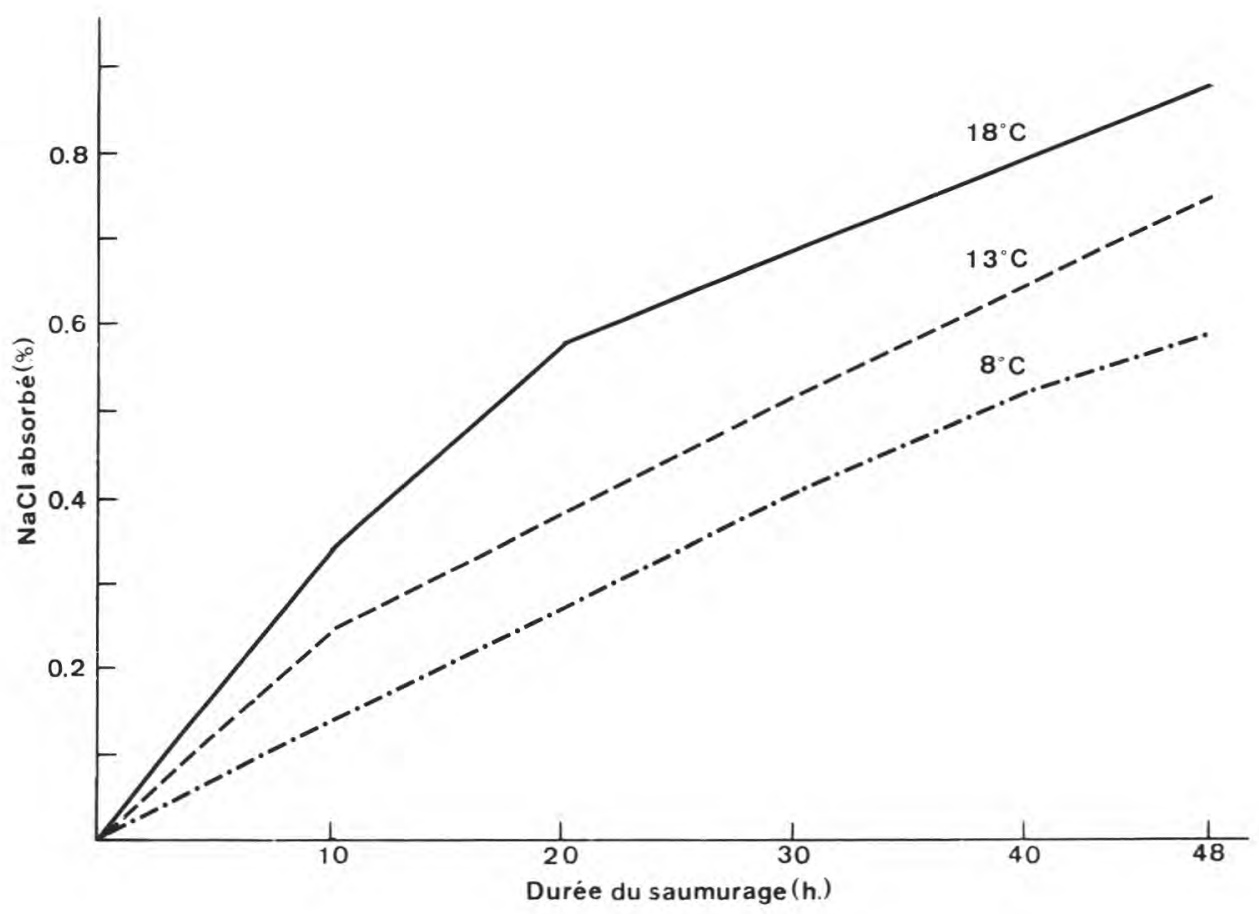

FIG. 4

Influence de la température de la saumure sur l'absorption du sel par l'Emmental. Brine temperature effect on Emmental cheese salt absorption. 
Si les pertes de poids apparentes ne sont pas très différentes selon la température de la saumure, on peut observer que les pertes sous forme de phase aqueuse sont sensiblement influencées. Dans les limites de température de ces observations, les échanges entre le fromage et la saumure sont d'autant plus grands que la température de la saumure est élevée puisque la perte de phase aqueuse est de $1,49 \%$ à $8{ }^{\circ} \mathrm{C}, 1,72 \%$ à $13{ }^{\circ} \mathrm{C}$ et $1,84 \%$ à $18^{\circ} \mathrm{C}$.

\section{E. Influence de la composition de la saumure}

\section{La concentration en $\mathrm{NaCl}$}

Pour étudier l'influence de ce facteur, nous avons utilisé les résultats de 39 essais obtenus à $48 \mathrm{~h}$ avec des saumures à $12-13{ }^{\circ} \mathrm{C}$ et à $\mathrm{pH} 5,1-5,5$ dont les concentrations en $\mathrm{NaCl}$ variaient de 204 à $318 \mathrm{~g} \cdot \mathrm{1}^{-1}$. Les courbes de la figure 5 représentent les cinétiques obtenues en regroupant, pour la clarté du graphique, ces essais en trois gammes de concentrations de saumure 200 à $249 \mathrm{~g} \cdot 1^{-1}, 250$ à $275 \mathrm{~g} \cdot 1^{-1}$ et plus de $275 \mathrm{~g} \cdot \mathrm{1}^{-1}$. L'augmentation de la concentration en $\mathrm{NaCl}$ de la saumure accroît la vitesse de l'absorption ainsi que la teneur en sel du fromage. Le calcul du coefficient de corrélation entre la concentration de la saumure et la quantité de sel absorbée et de la droite de régression donne les valeurs indiquées dans la figure 6.

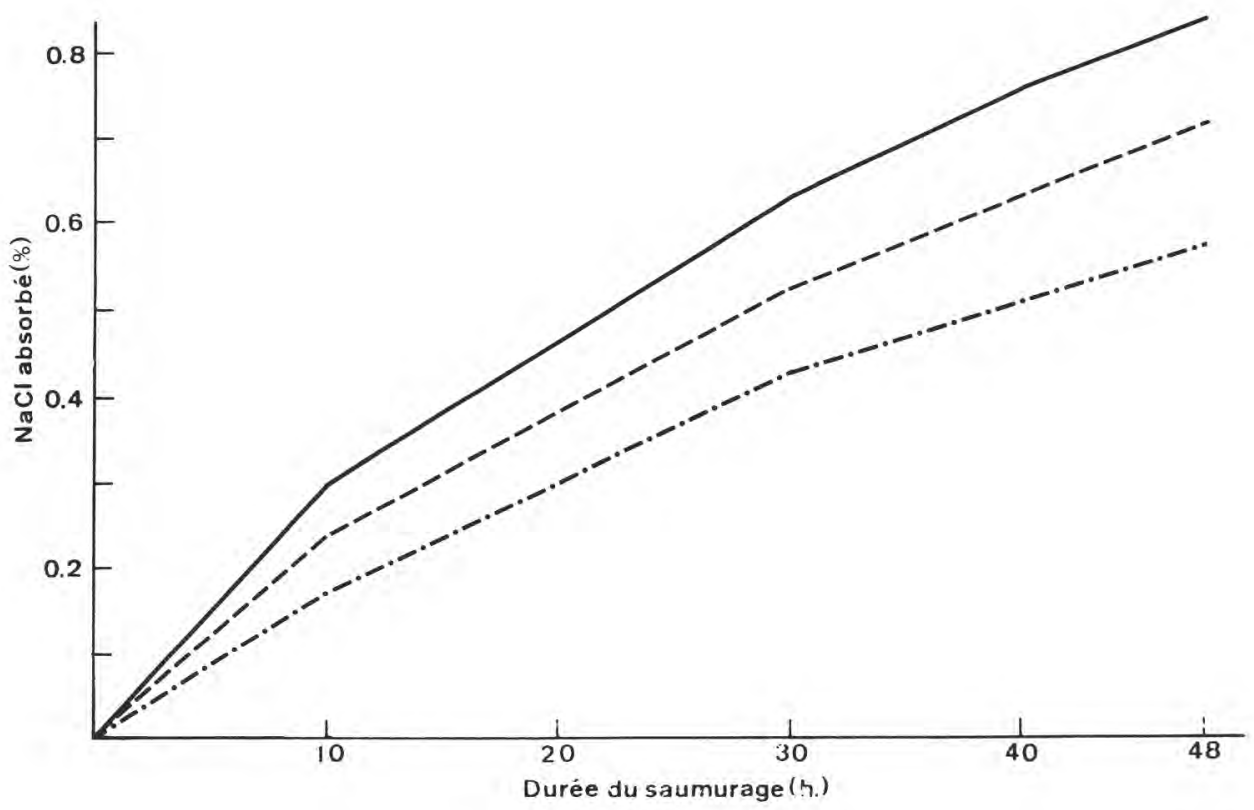

Fig. 5

Influence de la concentration de la saumure sur la cinétique de l'absorption du sel par l'Emmental.

Brine concentration effect on salt absorption kinetic by Emmental cheese.

--_-_. 200 à $249 \mathrm{~g} \cdot 1^{-1} ;----250$ à $275 \mathrm{~g} \cdot 1^{-1} ;-\ldots+$ de $275 \mathrm{~g} \cdot 1^{-1}$. 


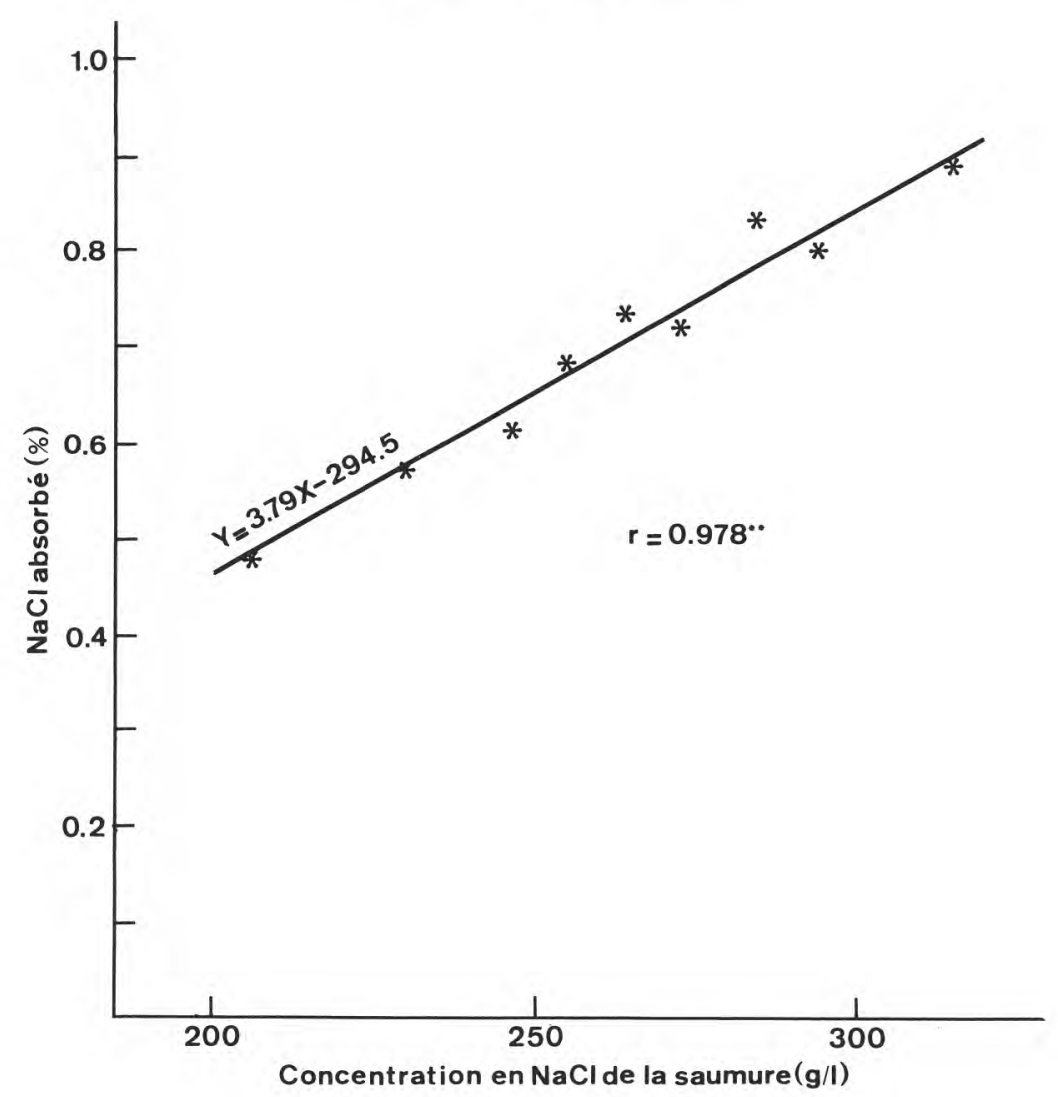

FIG. 6

Relation entre la concentration de la saumure et l'absorption du sel par l'Emmental.

Relationship between brine concentration and Emmental cheese salt absorption.

Cette relation hautement significative $(r=0,978)$ peut s'écrire :

sel absorbé en $48 \mathrm{~h}(\mathrm{~g} / 100 \mathrm{~kg})=3,79 \mathrm{Co} . \mathrm{S}-294,5$

où Co.S est la concentration de saumure en $\mathrm{g} \cdot \mathrm{l}^{-1}$.

L'autre observation que l'on peut effectuer concerne les pertes de poids au cours du saumurage, elles sont généralement d'autant plus fortes que la concentration de la saumure est élevée. Ainsi, plus la saumure est concentrée, plus les échanges entre celle-ci et le fromage sont importants.

2. Le $\mathrm{pH}$ de la saumure

Le pH d'une saumure fraîchement préparée est généralement de 6,5 à 7 . Par la suite, ce $\mathrm{pH}$ baissera au fur et à mesure de l'utilisation pour se rapprocher et se stabiliser à une valeur proche du $\mathrm{pH}$ du fromage saumuré. Dans ce travail, nous avons voulu isoler l'effet du seul $\mathrm{pH}$ en utilisant des 
saumures un petit nombre de fois (2 à 5), le $\mathrm{pH}$ de la saumure étant ajusté à l'acide lactique.

Pour établir l'influence de ce facteur, nous avons comparé 14 couples de fromages salés dans des saumures à $12-13^{\circ} \mathrm{C}$, de concentration en sel identique $\left(272 \mathrm{~g} \cdot 1^{-1}\right)$ mais dont le $\mathrm{pH}$ était compris soit entre 5,1 et 5,5 soit entre 6,2 et 7. L'évolution du $\mathrm{pH}$ de la saumure ne dépasse pas $0,1 \mathrm{pH}$ lors du salage d'un fromage. Lorsque nous utilisons une saumure fraîche non acidifiée, son $\mathrm{pH}$ de départ s'abaisse progressivement au cours des salages successifs. Le $\mathrm{pH}$ de la saumure utilisée le plus grand nombre de fois (5) a évolué ainsi : 6,706,$62 ; 6,62-6,54 ; 6,54-6,52 ; 6,53-6,49$.

La figure 7 indique clairement que le $\mathrm{pH}$ de la saumure n'a aucun effet sur l'absorption du sel et sur les pertes de poids.

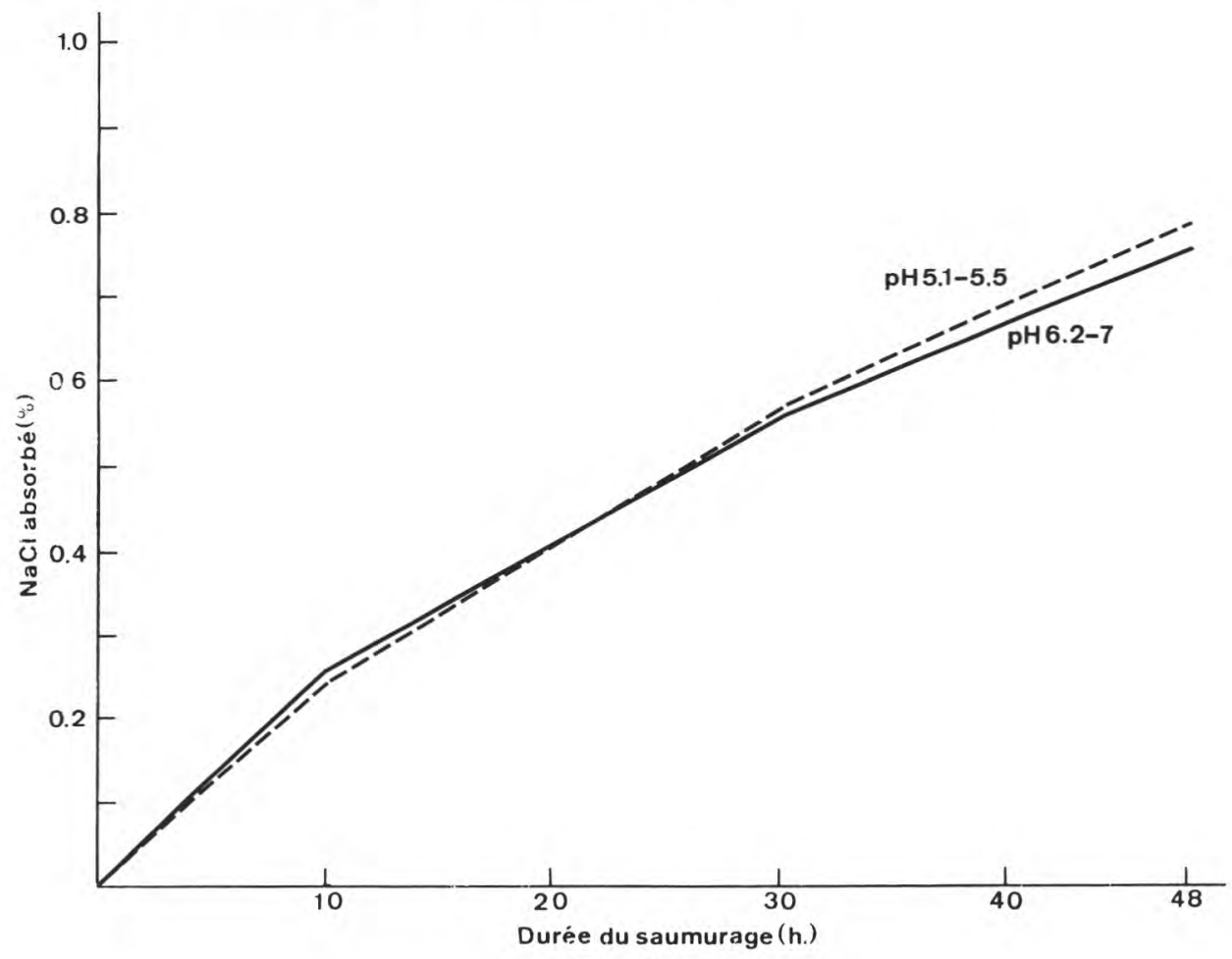

FIG. 7

Influence du $\mathrm{pH}$ de la saumure sur l'absorption du sel par l'Emmental.

Brine pH effect on Emmental cheese salt absorption.

\section{Teneur en lactosérum de la saumure}

$\mathrm{Au}$ fur et à mesure de son usage, la saumure s'enrichit en solutés provenant du fromage, et, du mélange d'eau et de sel qu'elle était au départ, la saumure devient progressivement une solution de sel dans la phase aqueuse 


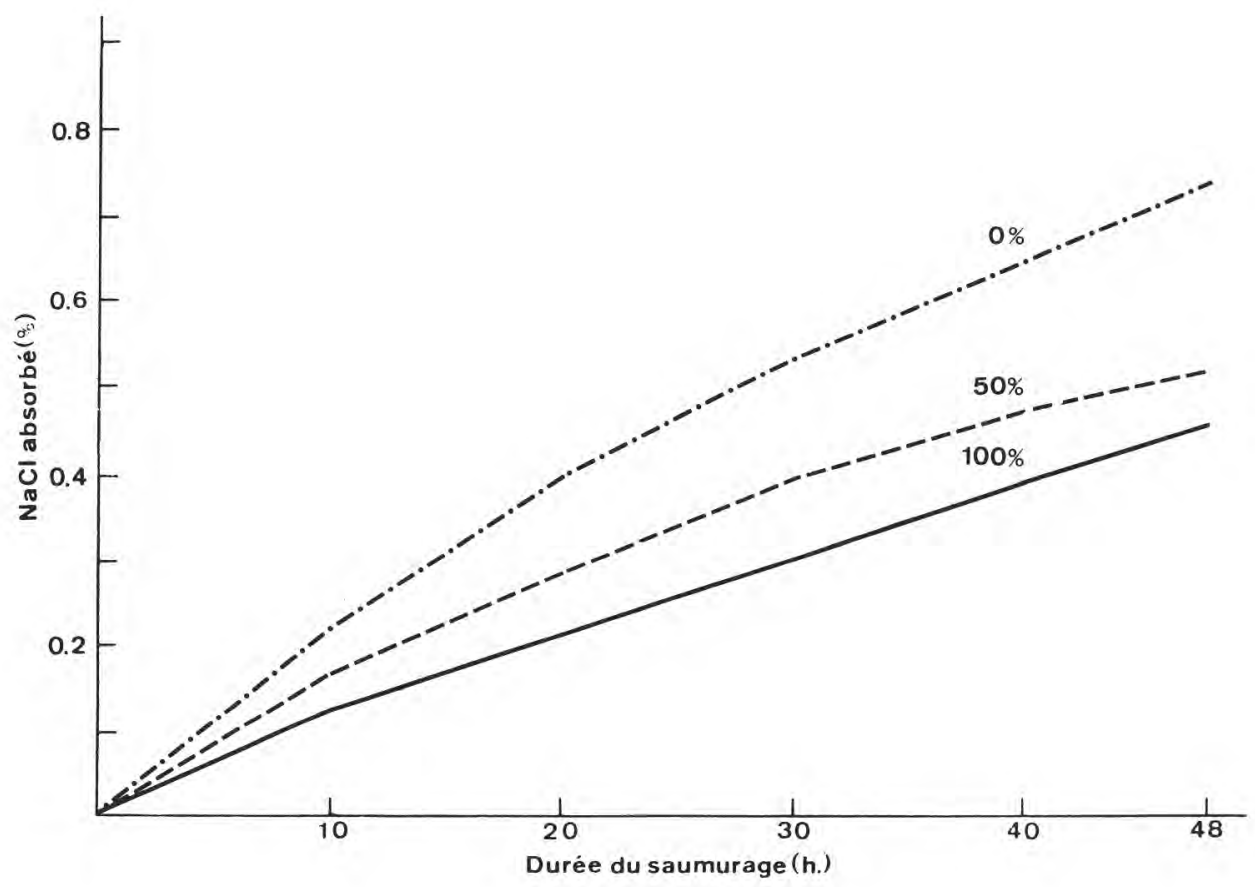

Fig. 8

Influence de la teneur en lactosérum de la saumure sur l'absorption du sel par l'Emmental. Teneur en lactosérum 0\%, $50 \%$ et $100 \%$.

Brine whey content effect on Emmental cheese salt absorption. Whey content : $0 \%, 50 \%$ and $100 \%$.

du fromage. Cette évolution se mesure généralement par l'augmentation de l'acidité de la saumure. Pour étudier cet effet, nous l'avons simulé en préparant une saumure à partir de lactosérums d'égouttage d'Emmental recueillis entre la deuxième et la sixième heure du pressage et une saumure contenant $50 \%$ de lactosérum, par dilution avec une saumure fraîchement préparée. Le pH des saumures était ajusté à 5,2-5,4 et les concentrations en sel étaient très voisines (266 à $\left.270 \mathrm{~g} \cdot \mathrm{1}^{-1}\right)$. Pour chaque teneur en lactosérum, 6 essais ont été effectués.

Les résultats obtenus (fig. 8) indiquent que l'absorption de $\mathrm{NaCl}$ par l'Emmental est meilleure lorsque la saumure est fraîchement préparée que lorsqu'elle contient beaucoup de lactosérum. La quantité de sel absorbée est réduite de $30 \%$ avec une saumure constituée de $50 \%$ de lactosérum et de $38 \%$ avec $100 \%$ de lactosérum. Si la différence d'absorption entre les deux saumures contenant du lactosérum est faible, par contre, les pertes en phase aqueuse par le fromage sont plus importantes : $1,22 \%(100 \%)$ contre 1,51\% $(50 \%)$, cette perte de phase aqueuse étant de $1,73 \%$ avec la saumure fraîche. 


\section{TABLEAU 4}

Absorption du $\mathrm{NaCl}$ et pertes de poids de l'Emmental en fonction de la durée de saumurage, de la température et de la composition de la saumure (moyennes)

Effects of brining time, temperature and brine compound on $\mathrm{NaCl}$ absorption and losses by Emmental cheese (means)

\begin{tabular}{|c|c|c|c|c|c|c|}
\hline \multicolumn{3}{|c|}{ Saumure } & \multirow{2}{*}{$\begin{array}{c}\text { Durée du } \\
\text { saumurage } \\
\text { H }\end{array}$} & \multirow{2}{*}{$\begin{array}{c}\mathrm{NaCl} \\
\text { absorbé } \\
\mathrm{g} / 100 \mathrm{~kg}\end{array}$} & \multirow{2}{*}{$\begin{array}{l}\text { Pertes de } \\
\text { poids } \\
\text { en } \%\end{array}$} & \multirow{2}{*}{$\begin{array}{l}\text { Nombre } \\
\text { d'essais }\end{array}$} \\
\hline $\mathrm{NaCl} \%$ & $t^{\circ}$ & $\mathrm{pH}$ & & & & \\
\hline 266 & $12-13^{\circ} \mathrm{C}$ & $5,1-7$ & 48 & 709,5 & 0,94 & 22 \\
\hline 267,5 & $12-13{ }^{\circ} \mathrm{C}$ & $5,1-7$ & 72 & 903 & 1,17 & 11 \\
\hline 264 & $12-13^{\circ} \mathrm{C}$ & $5,1-7$ & 96 & 1039 & 1,33 & 4 \\
\hline 290 & $8^{\circ} \mathrm{C}$ & $5,1-5,5$ & 48 & 579 & 0,91 & 5 \\
\hline 290 & $13^{\circ} \mathrm{C}$ & $5,1-5,5$ & 48 & 740 & 0,98 & 5 \\
\hline 292 & $18^{\circ} \mathrm{C}$ & $5,1-5,5$ & 48 & 874 & 0,97 & 5 \\
\hline 204 à 249 & $12-13^{\circ} \mathrm{C}$ & $5,1-5,5$ & 48 & 575 & 0,79 & 14 \\
\hline 250 à 275 & $12-13{ }^{\circ} \mathrm{C}$ & $5,1-5,5$ & 48 & 706 & 0,925 & 11 \\
\hline 275 à 318 & $12-13^{\circ} \mathrm{C}$ & $5,1-5,5$ & 48 & 834 & 1,04 & 14 \\
\hline 272 & $12-13^{\circ} \mathrm{C}$ & $5,1-5,5$ & 48 & 784 & 1,07 & 14 \\
\hline 272 & $12-13^{\circ} \mathrm{C}$ & $6,2-7$ & 48 & 784 & 1,07 & 14 \\
\hline 266 & $12-13^{\circ} \mathrm{C}$ & $(100)^{(1)}$ & 48 & 455 & 0,77 & 6 \\
\hline 270 & $12-13{ }^{\circ} \mathrm{C}$ & $(50)^{(2)}$ & 48 & 513 & 1,01 & 6 \\
\hline 268 & $12-13^{\circ} \mathrm{C}$ & $(0)^{(3)}$ & 48 & 732 & 1,00 & 6 \\
\hline
\end{tabular}

(1) (2) (3) Teneur en lactosérum de la saumure, en \%

(1) (2) (3) Whey content in the brine, \%.

\section{Discussion et conclusion}

Ce travail est basé sur la mesure indirecte de l'absorption du sel en observant la diminution de concentration de la saumure. Or, l'exsudation de la phase aqueuse du fromage peut interférer avec la mesure de l'absorption du sel et de sa cinétique du fait de la dilution de la saumure. Nous allons donc évaluer les conséquences de cet effet.

Normalement le bilan poids doit être équilibré : poids saumure départ = poids saumure fin + pertes de poids au cours du salage. Avec des saumures fraîches à $12-13^{\circ} \mathrm{C}$, le déficit moyen observé est de $0,305 \mathrm{~kg}$ pour des saumurages de $48 \mathrm{~h}$. Cette différence est toujours dans le même sens et conduit à penser qu'il y a une évaporation d'eau. L'augmentation de ce déficit lorsque la température de la saumure est portée à $18^{\circ} \mathrm{C}(+0,520 \mathrm{~kg})$, ou lorsque la durée du salage est de $72 \mathrm{~h}(+0,515 \mathrm{~kg})$ le confirme. Cette évaporation a pour conséquence de réduire l'abaissement de la concentration de la saumure. 
La dilution de la saumure et la prise de sel par le fromage conduisent à une diminution de la concentration de la saumure qui est l'un des facteurs importants agissant sur l'absorption du $\mathrm{NaCl}$. Les résultats obtenus permettent de calculer l'influence de cet effet. Ainsi, avec une saumure de départ à $23 \%$ de $\mathrm{NaCl}$, un poids de saumure de $130 \mathrm{~kg}$ et un Emmental de $75 \mathrm{~kg}$, nous obtenons en $48 \mathrm{~h}$ une absorption de $0,531 \mathrm{~kg}$ de $\mathrm{NaCl}$ et une perte de poids du fromage de $0,705 \mathrm{~kg}$. Compte tenu de l'évaporation $(0,305 \mathrm{~kg})$, la concentration de la saumure baisse de $4,78 \mathrm{~g} \cdot \mathrm{kg}^{-1}$. En utilisant l'équation de régression, liant la concentration de la saumure et l'Emmental, cela conduit à une diminution de l'absorption de $18 \mathrm{~g}$ soit $3,4 \%$.

Enfin, les résultats indiquent que le volume de la phase aqueuse exsudée par le fromage au cours du saumurage est plus important au début du salage. Le calcul basé sur l'évolution de la vitesse d'exsudation donne un volume exsudé de $1 \%$ en $24 \mathrm{~h}$ au lieu de $0,825 \%$ avec un calcul linéaire. Ainsi, le mode de calcul de la cinétique utilisé conduit à surestimer la vitesse d'absorption du sel puisque la dilution de la saumure contribue à l'abaissement de sa concentration. Pour un Emmental de $75 \mathrm{~kg}$, c'est une augmentation de $131 \mathrm{~g}$ de la phase solvante de la saumure, soit environ $0,1 \%$. A $24 \mathrm{~h}$, la surestimation de l'absorption du sel est donc de $23 \mathrm{~g}$ de $\mathrm{NaCl}$ avec un fromage de $75 \mathrm{~kg}$ et une saumure à $23 \%$ de $\mathrm{NaCl}$.

Cette étude n'avait, à notre connaissance, jamais été entreprise sur l'Emmental, seul Rothenbuhler (1970), l'avait effectuée, mais sur des disques de $5 \mathrm{~g}(\mathrm{~h}=2 \mathrm{~cm}$, diamètre $=1,8 \mathrm{~cm})$ dont la masse et le rapport surface/volume sont très éloignés d'une meule de 70 à $80 \mathrm{~kg}$. La vitesse d'absorption du sel décroît au cours du saumurage. Cette constatation avait déjà été effectuée par Jakubowski (1968) pour le Tilsit, par GeurTs et al. (1974) pour le Gouda et par HaRdY (1976) pour le Camembert.

L'absorption du sel que nous avons mesurée lors de ces essais est généralement plus élevée que celle observée par ailleurs dans de nombreuses fromageries lorsque l'on mesure la teneur en $\mathrm{NaCl}$ de l'Emmental (СнамвA, 1982). Cette plus grande absorption de sel par les fromages saumurés dans le bac expérimental a pour origine l'immersion complète du fromage et l'agitation permanente qui y est maintenue. HARDY (1976) explique l'influence de l'agitation par le maintien de la concentration en sel à l'interface fromage/saumure alors que dans une saumure statique cette concentration baisse du fait de l'absorption du sel et de la sortie de la phase aqueuse du fromage. Cette agitation doit également favoriser la diffusion dans la saumure des solutés provenant du fromage.

Nos résultats contredisent Huber (1977) qui n'a pas observé d'augmentation de la teneur en sel de l'Emmental lorsque le saumurage est prolongé de 3 à 4 jours. Par contre, Kurmann et al. (1976) ont obtenu une augmentation de $30 \%$ de la teneur en sel lorsque l'Emmental est saumuré quatre jours au lieu de deux jours. Cette augmentation est inférieure à celle que nous avons obtenue $(+61,5 \%)$, mais les fromages n'étaient pas complètement immergés dans la saumure et il est probable que l'humidité de la zone corticale des Emmentals suisses soit plus faible que celle des Emmentals utilisés pour ce travail. En effet, Kurmann et al. (1976) indiquent que le pressage en moule métallique de leurs fromages durait $22 \mathrm{~h}$ au lieu de $8 \mathrm{~h}$ pour les nôtres. Leur 
saumure contenait $23 \%$ de $\mathrm{NaCl}$ soit la même concentration que la nôtre, mais ils ne précisent pas si elle était fraîchement préparée.

La perte de poids apparente du fromage lors du saumurage ne traduit que partiellement l'exsudation de la phase aqueuse du fromage. Elle est, en fait, égale à la somme perte de poids + absorption de sel. Le rapport exsudation/ gain de sel est de 2,32 à $48 \mathrm{~h}, 2,29$ à $72 \mathrm{~h}$ et 2,28 à $96 \mathrm{~h}$. Ces valeurs sont plus proches de celles obtenues par GeurTs (1974) pour le Gouda que celles mesurées par HARDY (1976) avec le Camembert : respectivement 2,5 et 1,7.

La composition de cet exsudat est, selon HARDY (1976), très proche de celle d'un lactosérum de fin d'égouttage. Toutefois, cela ne semble se vérifier qu'avec une saumure fraîchement préparée. En effet, les observations effectuées avec des fromages à pâte molle par LACRAMPE et al. (1971) sur des saumures industrielles établissent que les pertes de substances azotées (58 mg par $\mathrm{kg}$ de fromage) sont essentiellement constituées d'azote non protéique : 70 à $75 \%$, ce qui est une proportion très différente de celle du lactosérum. De plus, les comparaisons effectuées avec du Camembert par TERRE et al. (1985) entre saumures « vierges " et « usées " montrent que les échanges de solutés entre le fromage et la saumure dépendent de la composition de cette dernière. Les résultats que nous avons obtenus par ailleurs (CHAMBA, 1982) en observant l'évolution de la composition de saumures d'Emmental pendant 150 jours indiquent aussi que le transfert de chaque soluté évolue au cours du vieillissement de la saumure. Ainsi, alors que l'évolution de la teneur en lactate de la saumure est quasiment linéaire, les pertes de calcium sont de $54 \mathrm{mg} \cdot \mathrm{kg}^{-1}$ d'Emmental lors du premier mois d'utilisation de la saumure et ne sont plus que de $2,9 \mathrm{mg} \cdot \mathrm{kg}^{-1}$ au cours des quatre mois suivants. Ce mécanisme est également observé par Terre et al. (1985). La composition de la phase aqueuse exsudée par l'Emmental au cours du saumurage dépend donc étroitement du degré «d'usure» de la saumure.

La température de la saumure joue un rôle évident ; par comparaison à une saumure à $13{ }^{\circ} \mathrm{C}$ la quantité de sel absorbée est de $78,2 \%$ à $8{ }^{\circ} \mathrm{C}$ et de $118 \%$ à $18{ }^{\circ} \mathrm{C}$. Ces résultats sont en accord avec ceux de nombreux auteurs obtenus sur des fromages aussi différents que le fromage blanc affiné en saumure (MANSOUR, 1972), le Gouda (GeurTs et al., 1974), le Camembert (Hardy, 1976), le Grana (Bottazzi, 1967). En Emmental, Oehen (1971) sans donner de valeurs chiffrées indique que l'absorption du sel est très lente en dessous de $10^{\circ} \mathrm{C}$; il en est de même de Huber (1976) selon lequel une température de la saumure de $16^{\circ} \mathrm{C}$ conduit à une teneur en sel plus forte qu'à $12^{\circ} \mathrm{C}$. Kurmann et al. (1976) ont saumuré des Emmentals à $1{ }^{\circ} \mathrm{C}, 15^{\circ} \mathrm{C}$ et $30^{\circ} \mathrm{C}$, l'analyse des fromages affinés a donné les teneurs en $\mathrm{NaCl}$ suivantes : respectivement $0,46 \%, 0,52 \%$ et $0,65 \%$. Ces données sont en accord avec nos résultats même si ces températures de saumure ne doivent pas se rencontrer dans la pratique.

Si les pertes de poids du fromage ne sont pas fortement influencées par la température de la saumure, en revanche, l'exsudation de la phase aqueuse du fromage l'est nettement. Ainsi, une température trop basse limite les échanges entre la saumure et le fromage, ce qui réduit l'importance du complément d'égouttage qu'apporte le salage.

La concentration en sel de la saumure influence l'absorption du sel par l'Emmental. C'est un effet général pour tous les fromages salés pendant 
quelques heures ou quelques jours (JAKUBOWSKI, 1968 ; GeURTS et al., 1974 ; HARDY, 1976 ; Huber, 1976). Les essais effectués avec des saumures à $12-13^{\circ} \mathrm{C}$ dont la concentration variait de $204 \mathrm{~g} \cdot 1^{-1}$ nous ont permis d'obtenir la relation suivante :

sel absorbé en $48 \mathrm{~h}(\mathrm{~g} / 100 \mathrm{~kg})=3,79$ concentration saumure $\left(\mathrm{g} \cdot \mathrm{l}^{-1}\right)-294,5$ $\left(\mathrm{r}=0,978^{* *}\right)$.

Ces résultats sont en accord avec ceux obtenus par d'autres auteurs et indiquent que l'Emmental se comporte comme de nombreux autres fromages. L'effet de la concentration en $\mathrm{NaCl}$ de la saumure est général, plus elle est concentrée, plus l'absorption de sel est forte, comme le montre la formule établie par JAKUBOWSKI (1968) pour le Tilsit :

$$
\mathrm{Y}=\frac{\mathrm{C}}{1 \cdot \frac{1}{\mathrm{D} \sqrt{\mathrm{t}}}}
$$

$\mathrm{Y}$ : concentration en sel $(\%)$ du fromage

$\mathrm{C}$ : concentration de la saumure $(\%)$

$\mathrm{t}$ : durée du saumurage (sec.)

D est une constante de diffusion propre à chaque fromage qui tient compte de la surface relative du fromage.

Il en est de même pour les fromages à pâte molle et Hardy (1983) se basant sur la loi de FICK des transferts de matière par diffusion obtient la formule suivante :

$$
\mathrm{S}=2 \mathrm{C}_{\mathrm{s}} \cdot \frac{\mathrm{A}}{\mathrm{V}} \cdot\left[\frac{\mathrm{Dt} \mathrm{s}_{\mathrm{s}}}{\pi}\right] \frac{1}{2}
$$

où $\mathrm{S}=\% \mathrm{NaCl}$ absorbé dans l'eau du fromage

$\mathrm{C}_{\mathrm{s}}=$ concentration de la saumure ( $\% \mathrm{NaCl}$ dans l'eau)

$A=$ surface du fromage $\left(\mathrm{cm}^{2}\right)$

$\mathrm{V}=$ volume du fromage $\left(\mathrm{cm}^{3}\right)$

$D=$ coefficient de diffusion du sel $\left(\mathrm{cm}^{2} \cdot \mathrm{j}^{-1}\right)$

$\mathrm{t}_{\mathrm{s}}=$ durée du saumurage en jours.

En Gouda, GeurTs et al. (1974) a également vérifié l'application de la loi de FICK dans laquelle la différence de concentration du sel dans l'eau de la saumure et dans l'eau du fromage explique l'absorption du sel par le fromage.

Sans donner de valeurs, Huber (1977) indique que la teneur en sel de l'Emmental est plus forte lorsque la saumure contient $25 \%$ de $\mathrm{NaCl}$ au lieu de $21 \%$. Toutefois, BotTazzi (1967) signale que l'absorption du sel est meilleure en Parmesan à $21 \%$ qu'à $25 \%$ de sel dans la saumure. Ce phénomène a également été observé par Mansour (1972) avec des fromages conservés en saumure pendant plusieurs mois. L'explication donnée par ces deux auteurs est que les pertes d'eau sont plus importantes avec des saumures plus concentrées ce qui conduit à la formation d'une croûte plus sèche et, en conséquence, à un ralentissement de la pénétration du sel. L'Emmental n'est pas saumuré assez longtemps pour qu'un tel phénomène soit observé, bien que les pertes de poids du fromage soient plus fortes lorsque la concentration de la saumure augmente. 
Nos résultats indiquent que le salage dans une saumure à $300 \mathrm{~g} \cdot 1^{-1}$ au lieu de $200 \mathrm{~g} \cdot 1^{-1}$ augmente la teneur en sel de $77 \%$, alors que pour la même augmentation de concentration de la saumure, HARDY (1976) obtient $+141 \%$. C'est très probablement la différence d'humidité ou plus exactement d'eau solvante entre ces deux fromages qui explique ce comportement. En effet, en se basant sur une proportion de 0,10 à $0,15 \mathrm{~g}$ d'eau non solvante du $\mathrm{NaCl}$ par $\mathrm{g}$ de protéine (GeurTs et al., 1974), le Camembert contient entre 51 et $53 \%$ d'eau solvante contre 30,5 à $32 \%$ pour l'Emmental (zones corticales). Ainsi, l'Emmental a une capacité d'absorption du sel (en $\mathrm{g}$ pour $100 \mathrm{~g}$ de fromage) voisine des $2 / 3$ de celle du Camembert.

Sur le $\mathrm{pH}$ de la saumure, les résultats publiés sont beaucoup plus rares. Hardy (1976) a observé que le pH agit peu sur la quantité de sel absorbé par le Camembert et qu'un abaissement du $\mathrm{pH}$ de 3,9 à 1,5 augmente sensiblement les pertes d'humidité. Cet effet est beaucoup plus faible lorsque le $\mathrm{pH}$ de la saumure utilisée est de 3,9 au lieu de 6 . Au contraire, les pertes en solutés du Camembert sont influencées par le pH de la saumure. Ainsi, Terre et al. (1985) montrent qu'à $\mathrm{pH} 7$, les pertes en acide lactique, en potassium et surtout en calcium sont plus fortes qu'à $\mathrm{pH} 4,6$; le $\mathrm{pH}$ de la saumure n'ayant pas d'influence sur la migration du phosphore. En Emmental, le pH de la saumure n'a aucune influence sur l'absorption du sel et les pertes de poids, tout au moins pour des valeurs variant de 5,1 à 7 . Le vieillissement des saumures, c'est-à-dire l'accumulation des solutés provenant du fromage, gêne les échanges entre le fromage et la saumure (KeIlling, 1968 ; LACrampe et al., 1971 ; MAYr, 1976). L'hypothèse la plus vraisemblable pour expliquer cette réduction de l'absorption du sel se base sur la forte diminution, voire l'absence de transferts de solutés (acide lactique, calcium, potassium, azote non protéique) entre le fromage et les saumures contenant du lactosérum, comme l'ont observé Terre et al. (1985) avec une saumure industrielle "usagée". A l'inverse, dans une saumure «vierge », le transfert des solutés est intense et rapide et il peut être assimilé, pour une part, à un «lavage » de la zone corticale du fromage. De ce fait, la quantité d'eau solvante de la phase aqueuse de la zone corticale du fromage est augmentée ce qui accroît l'absorption du sel. De plus, la perte de calcium par le fromage facilite probablement la fixation de sel par la paracaséine, elle atteindrait $0,08 \mathrm{~g}$ par $\mathrm{g}$ de paracaséine (HARDY, 1983), limitant ainsi la réduction du gradient de concentration en $\mathrm{NaCl}$ entre l'eau solvante du fromage et la saumure.

Ainsi, pour maintenir constante l'efficacité de la saumure, outre le maintien de la concentration en $\mathrm{NaCl}$, il y aurait intérêt à effectuer soit des renouvellement partiels et réguliers avec une saumure neuve, soit une ultrafiltration comme le proposent TERRE et al. (1985) afin d'assurer l'élimination des solutés minéraux et organiques provenant des fromages. Avec le remplacement par de la saumure neuve des pertes et du rétentat éliminé $(0,6 \%$ à $1 \%)$, l'ultrafiltration, conduisant à une épuration microbienne quasi totale, devrait permettre une utilisation de longue durée des saumures.

Enfin, l'EST du fromage, ou plus exactement celui de la zone corticale, intervient nettement : plus cette zone est humide, plus la quantité de sel absorbée sera grande. Le coefficient de corrélation que nous avons obtenu entre l'humidité et la teneur en sel du fromage est de 0,85 (hautement significatif). Nous retrouvons donc sur l'Emmental l'influence de l'humidité du 
fromage qu'avaient montrée Geurts et al. (1974) avec le Gouda lorsqu'ils avaient déterminé le coefficient de pseudo-diffusion du sel dans l'eau du fromage. Ce coefficient $\left(\mathrm{cm}^{2} \cdot \mathrm{j}^{-1}\right)$ varie dans le Gouda de 0,10 à 0,24 lorsque l'humidité du fromage passe de $36,2 \%$ à 46,7 \%. HARDY (1976) a trouvé 0,22 pour le Camembert. Morris et al. (1985) classant les coefficients de pseudodiffusion du sel de différents fromages en fonction de leur humidité donnent 0,14 pour l'Emmental, 0,15 pour le Cheddar, 0.16 pour le Jarlsberg, 0,19 pour le Romano et 0,22 pour le Bleu. La dessiccation de l'une des faces du fromage alors que l'autre est en contact avec un foncet généralement humide peut expliquer les différences de teneur en sel que nous avons observées entre les deux faces de certains Emmentals (СнамвA, 1982).

En conclusion, on peut dire que l'absorption du sel par l'Emmental dépend de trois groupes de facteurs :

- La composition du fromage et en particulier de l'humidité de sa zone corticale. En pratique, il faut donc éviter le dessèchement de la surface de l'Emmental avant le saumurage afin de favoriser l'absorption du sel.

- De la durée du saumurage.

- Des caractéristiques de la saumure : concentration en sel, degré de vieillissement, température. L'agitation de la saumure étant un moyen de maintenir constantes les caractéristiques de la saumure en contact avec le fromage. La maîtrise de chacun de ces facteurs permet d'assurer un salage efficace et régulier.

Reçu le 26 novembre 1986 Accepté pour publication le 4 décembre 1987

\section{Remerciements}

Nous remercions $M$. Charles ALAIS qui dès l'origine et tout au long de ce travail nous a accueilli, guidé et conseillé.

\section{Références bibliographiques}

AlaIS C., 1975. Science du Lait, Principes des Techniques Laitières. $3^{\text {e }}$ édition, Ed. SEPAIC, Paris.

Bergere J.L., Rousseaux P., Ducruet P., Mocquot G., Hermier J., Gouet P., Zelter Z.S., 1969. Expérimentation sur la fabrication d'Emmental et de Comté avec du lait de vaches nourries à l'ensilage AIV. I. Aspects technologiques, Bull. Tech. Inf., 239, 1-30.

Botrazzi V., 1967. Ricerche sulla microbioligia del formagio Grana. VI parte : Gonfiore centrale ; assorbimento et diffusion del cloruro di soda nelle pasta. Sci. Tec. Latt. Casearia, 18, 363-382.

Boy G.J., 1969. La teneur en sel des fromages. Tech. Lait., 628, 17.

Снамва J.F., 1982. Le salage de l'Emmental - étude de l'absorption, de la migration, de la répartition du sel et de son influence sur la qualité du fromage. Thèse Université Nancy $\mathrm{I}$.

Geurts T.J., Walstra P., Mulder H., 1974. Transport of salt and water during salting of cheese. 1. Analysis of the processes involved. Neth. Milk Dairy J., 28, 102-129.

Grappin R., 1976. Guide pour l'évaluation des méthodes d'analyses de routine. Lait, 51, 608-621. 
HARDY J., 1976. Etude de la diffusion du sel dans les fromages à pâte molle de type Camembert. Comparaison du salage à sec et du salage en saumure. Thèse Docteur-Ingénieur, Université Nancy I.

HARDY J., 1983. Diffusion et distribution du chlorure de sodium dans les fromages. Influence sur l'activité de l'eau et les propriétés de sorption de l'eau. Thèse de Docteur es Sciences, Institut National Polytechnique de Lorraine.

HubER A., 1977. Einfluse des Salzbades auf die qualität von Hart-und Schnittkäse. Milchwirtsch. Ber., 53, 271-279.

JAKUBOWSKI J., 1968. Untersuchungen über die Kinetik der diffusion von Salz im Käse. Milchwissenschaft, 23, 282-287.

KeIlling J., 1968. Le vieillissement des saumures dans les fromageries de Gruyère et d'Emmental. Tech. Lait., 580, 12-13.

Kosikowski F.V., Mocquot G., 1958. Progrès de la Technologie Fromagère. Ed. FAO.

Kurmann J.L., Gehriger G., Steffen Ch., Kaufmann H., 1967. Die Salzbadbehandlung der Emmentaler bei verschiedenen temperaturen. Schweiz. Milchztg., 9, 57-58.

Lacrampe J.L., Hardy J., Ramet J.P., Weber F., 1971. Contribution à l'étude de l'évolution chimique et du traitement des saumures de fromagerie. Lait, 51, 158-175.

Le Graet Y., Brulé G., Maubois J.L., Oeuvrard G., 1986. Répartition et évolution des éléments minéraux au cours de l'affinage des fromages à pâte cuite type Beaufort. Lait, $66(4), 391-404$.

Mair Waldburg H., Heinrich C, 1966. Zum Kochsalzgehalt des emmentalers. Disch. Molk. Ztg., $26,1142-1147$.

Mansour A., Alais C., 1971. Le mécanisme du salage des fromages en saumure. Rev. Lait. Fr., 290, 641-645.

Mansour A., 1972. Etude biochimique et microbiologique du fromage affiné en saumure. Thèse de Docteur Ingénieur Nancy I

MARTin M., 1898. La maturation des fromages. Congrès Exposition de Laiterie Poligny.

MAYr A., 1976. Zur kochsalzkonzentration in Emmentalerkäse. Dtsch. Molk. Ztg., 17, 493-496.

Morris H.A., Guinee T.P., Fox P.F., 1985. Salt Diffusion in Cheddar Cheese. J. Dairy Sci., 68, $1851-1858$.

MUNier J.B., 1858. Manuel des fromageries ou traité de la fabrication des fromages de gruyère Septmoncel, chevrets, Mont d'Or, etc. Imp. Lithographique F. Gauthier, Lons-le-Saunier (bibliothèque INRA Poligny).

OEHEN V., 1969. New gärungstechnische Erkenntnise. Schweiz Milchztg., 421-423.

Terre E., Le Graet Y., Brulé G., Maubois J.L., 1985. Etude du transfert des solutés des fromages à pâte molle dans les saumures. Intérêt du traitement par ultrafiltration sur membrane. Tech. Lait., 997, 39-47.

Uotila H., 1965. Uber die Unterschiede im Gebalt und Trockensubstanz Felt und $\mathrm{NaCl}$ des finnischen Emmentaler an vessechiedenen Stellen des Käselaibes. Finish J. Dairy Sci., 25, 3-11.

Von Roethenbuhler E., 1970. Der kochsalzgehalt des Emmentalerkäse heute, im Vergleich zu früher. Schweiz. Milchztg., 1047-1051. 